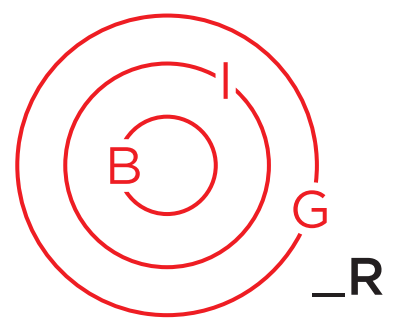

ART \& BORDERS

En français

\title{
Murs frontières et virtualité
}

\section{Hélène Mutter}

Dans le cadre de la section spéciale Art \& Borders, l'artiste plasticienne Hélène Mutter interroge la perception des frontières étatiques par le biais de la post-photographie. Depuis l'accessibilité technologique de Google Earth, le flou des séparations entre les nations jurent avec l'appréciation physique et vécue du passage. On assiste à un aplatissement des réalités géopolitiques. L'artiste propose pour BIG_Review cet article inédit sur son projet photographique "Lines » qui avait été exposé au festival Fotolimo dont un article dédié est dans ce même numéro présenté.

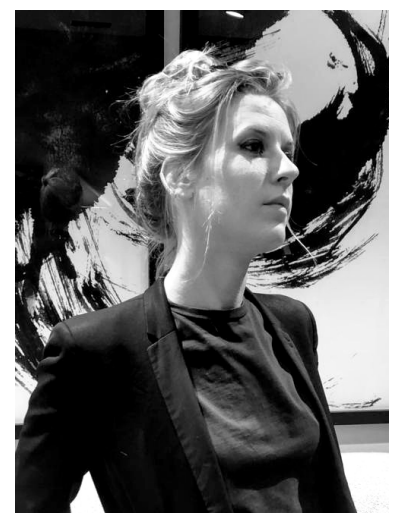

www.helenemutter.com

\section{A propos de l'artiste}

Artiste plasticienne, docteure en Art et Sciences de l'Art, chercheuse, Hélène Mutter soutient en 2020 sa thèse de doctorat intitulée "La guerre à l'épreuve de l'image-Art et dispositifs visuels ", réalisée en collaboration avec l'Académie Royale des Beaux-Arts et l'Université Libre de Bruxelles (ULB).

Sensible aux transformations politiques et sociales du monde qui nous entoure via une approche qui est celle de l'image au sens large, Hélène Mutter interroge les façons que nous avons de documenter le monde, et particulièrement les situations de crise et de conflit, par la création d'images, d'archives et de visuels, qu'ils soient produits par l'ingénierie militaire, des logiciels ou diffusés dans les médias.

Fin 2019, elle se rend au Liban alors en pleine révolution, afin d'y effectuer un projet photographique dans le cadre de sa résidence à la Beirut Art Residency. Son travail a récemment été publié dans la revue numérique Humanities, Arts and Society initiée par UNESCO-MOST, et elle est lauréate, en 2021, de la résidence artistique au Centre Culturel de Rencontre de Neimenster à Luxembourg.

Elle est également collaboratrice scientifique de la Faculté de Philosophie et Sciences Sociales de l'Université Libre de Bruxelles, laboratoire Repi (Recherche et Études en Politique Internationale). 
Borders in Globalization Review | Volume 3 | Issue 1 | Fall/Winter 2021

Mutter, "Murs frontières et virtualité"

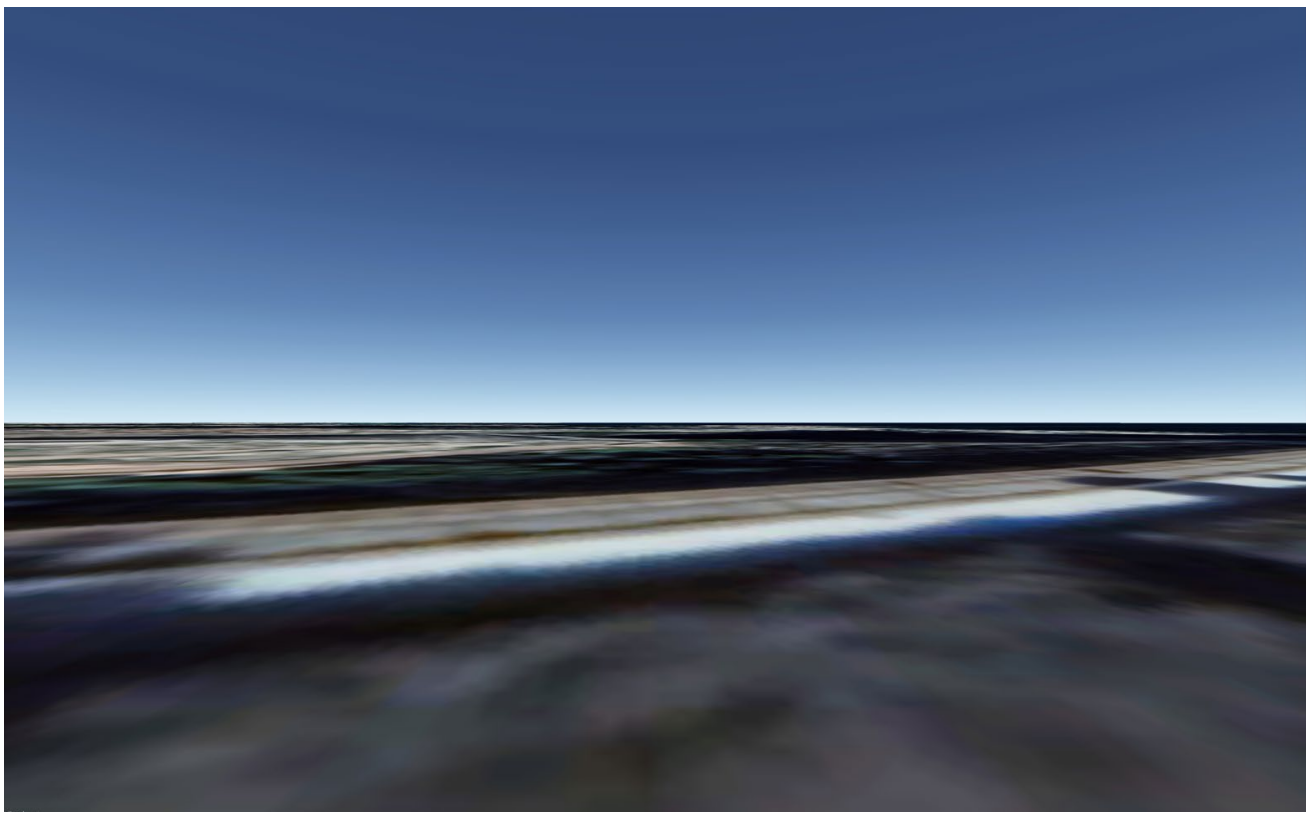

(C) 2018 Google

\section{Introduction}

De moins en moins vue au travers de sa fonction de représentation de la réalité, l'image photographique devient abstraite, non-figurative, un objet technique en lui-même et pour lui-même. Nous reviendrons, dans cette contribution, sur des questionnements précédemment soulevés dans mon travail de thèse qui a porté, à partir de l'analyse et de l'étude d'archives photographiques de la première guerre du Golfe de 1991, sur les différents régimes de visibilité mis à l'œuvre dans les représentations actuelles des conflits. Ma réflexion s'est portée sur les images produites au sein même de l'armée, afin de proposer une alternative visuelle face aux dangers liés à l'esthétisation des images de guerre. En opérant un décentrement du regard face à ces images, il s'est agi de penser en termes de "dé- subjectivation » où l'ensemble des dispositifs visuels mis en œuvre dans le cadre des conflits participe, de fait, à la dé-subjectivation du regard. Avec l'arrivée de la photographie sur le champ de bataille, c'est tout le champ de perception de la guerre qui se transforme. Questionner l'image produite dans le contexte de la guerre, c'est donc à la fois questionner son champ de perception et les différentes trajectoires de visibilité qui composent son environnement visuel. La guerre doit être pensée en lien avec notre regard. Depuis la guerre du Golfe, conflit qui a marqué une rupture dans la façon de penser et de représenter la guerre, le monde est devenu un écran géant sur lequel se succèdent, et de façon de plus en plus rapide, un flux et un flot d'images ininterrompus. L'image de guerre, telle qu'elle était pensée, montrée, imaginée jusque-là, n'allait plus jamais être la même. L'arrivée de la vue aérienne dans le champ de la guerre, la transformation du regard et l'abandon du corps produisent une image qui ouvre les limites visuelles auxquelles nous étions habitués jusqu'alors. La question du processus de production de l'image photographique soulève un questionnement qui n'est pas sans lien avec la dé-subjectivation à l'œuvre dans le champ de la guerre. L'opérateur placé derrière son appareil photo produit quelque chose " avec une machine », tout comme l'ingénierie militaire produit méthodiquement et logiquement sa propre imagerie. II s'agit alors de penser le renseignement et la reconnaissance aérienne militaire en termes de " globalisation de l'emploi de la puissance aérienne » (Aubout 2011, 89), où la guerre se pense aussi dans une troisième dimension.

Aujourd'hui, et accessible à tous, le logiciel Google Earth est devenu " l'œil du ciel », incarnant la figure contemporaine de la vision panoptique. En quelques clics, n'importe quel utilisateur se retrouve tel Icare survolant le monde, parcourant en une fraction de seconde, ou presque, la totalité du globe. C'est à partir d'un point de vue identique à cet œil absolu que le militaire, derrière son écran, aura droit de vie et de mort sur les populations. Selon cette perspective, nous pouvons affirmer que la logique de qui utilise Google Earth devient la même que celle développée par l'armée : n'importe qui peut survoler l'ensemble d'un territoire sans même s'en approcher, tout en étant en réalité situé à des milliers de kilomètres, derrière son écran d'ordinateur. 


\section{Dominer le monde}

La question de la distance, de la bonne distance, est une chose importante, à la fois pour voir, mais aussi pour toucher ou être touché. Tenir la distance permet d'un point de vue stratégique de dicter le rythme du combat tout en évitant de se faire toucher. Ni trop loin ni trop près. Dans les guerres napoléoniennes, on recommandait aux soldats de ne pas tirer avant de voir le blanc des yeux de l'adversaire. La distance s'est considérablement allongée depuis. Mais la violence de l'impact, elle, a peu changé. À travers les nouvelles technologies employées par les militaires via les dispositifs de prise de vue (drones, camérassuicides, images satellites), il est intéressant d'observer que l'articulation proximité/distance est au cœur même de la notion de combat. Qu'elle soit horizontale lors de déplacements latéraux ou frontaux entre des adversaires, ou verticale du point de vue d'une attaque aérienne, la distance multiplie les intervalles et l'espace qu'il reste à parcourir avant la frappe, l'impact. Dans la guerre qui en passe par les nouvelles technologies, le soldat derrière son écran de contrôle apparaít ici comme l'opérateur d'une guerre qui se joue aujourd'hui sans corps à corps. Les temps de l'assaut et du combat sont réduits à l'instant de la frappe de la cible, et la précision dite « chirurgicale » est censée provoquer des dégâts sans causer de dommages collatéraux. Dès lors, à quelle distance se situer ? À quel moment attaquer, abolir la distance qui sépare et/ou rapproche deux adversaires ? Cette violence maîtrisée, ritualisée, est bien synonyme de mort. "Le spectacle d'êtres humains luttant l'un contre l'autre, quelle qu'en soit la raison, est excessivement perturbant », écrit Joyce Carol Oates dans son ouvrage De la boxe. Avec vingt-cinq siècles d'écart, nous voici devant une nouvelle application de l'art de la guerre : soumettre l'ennemi sans combat (Tzu
2008, 142) ? L'apparition de la reconnaissance aérienne sur le champ de bataille, puis de la photographie aérienne comme document d'expertise au sein du monde militaire participe, de fait, à ce changement d'échelle dans les stratégies modernes de combat. Médium profondément séduisant et toujours très présent dans notre imaginaire, les vues d'en haut engendrent des changements perceptifs considérables. La vue aérienne fait basculer notre regard d'Homme en un regard voulu objectif et neutre, transformant la surface terrestre en écran. En effet, l'espace visuel devient global et le regard souverain est absolu.

La vue aérienne, grâce aux technologies contemporaines, permet une approche globale des environnements dans lesquels nous vivons, transformant notre regard et la vision que nous avions du monde et construisant un espace désormais entièrement franchissable. Dans un monde moderne en pleine expansion urbanistique et au devenir machinique grandissant, les innovations visuelles sont nombreuses et il est question de « détecter un au-delà de la surface optique » (Frizot 2001, 387).

\section{À propos de Google Earth}

Depuis la première photographie de la Terre prise de l'espace datée au 24 octobre 1946 et la première image de la Terre en son entier véhiculée par la NASA en 1966, l'imagerie spatiale n'a cessé de se développer jusqu'à nous représenter la Terre comme un globe virtuel (Coulais $2015,175)$ sur notre ordinateur. Mais à partir des années 1960 et l'envoi du premier homme dans l'espace, c'est

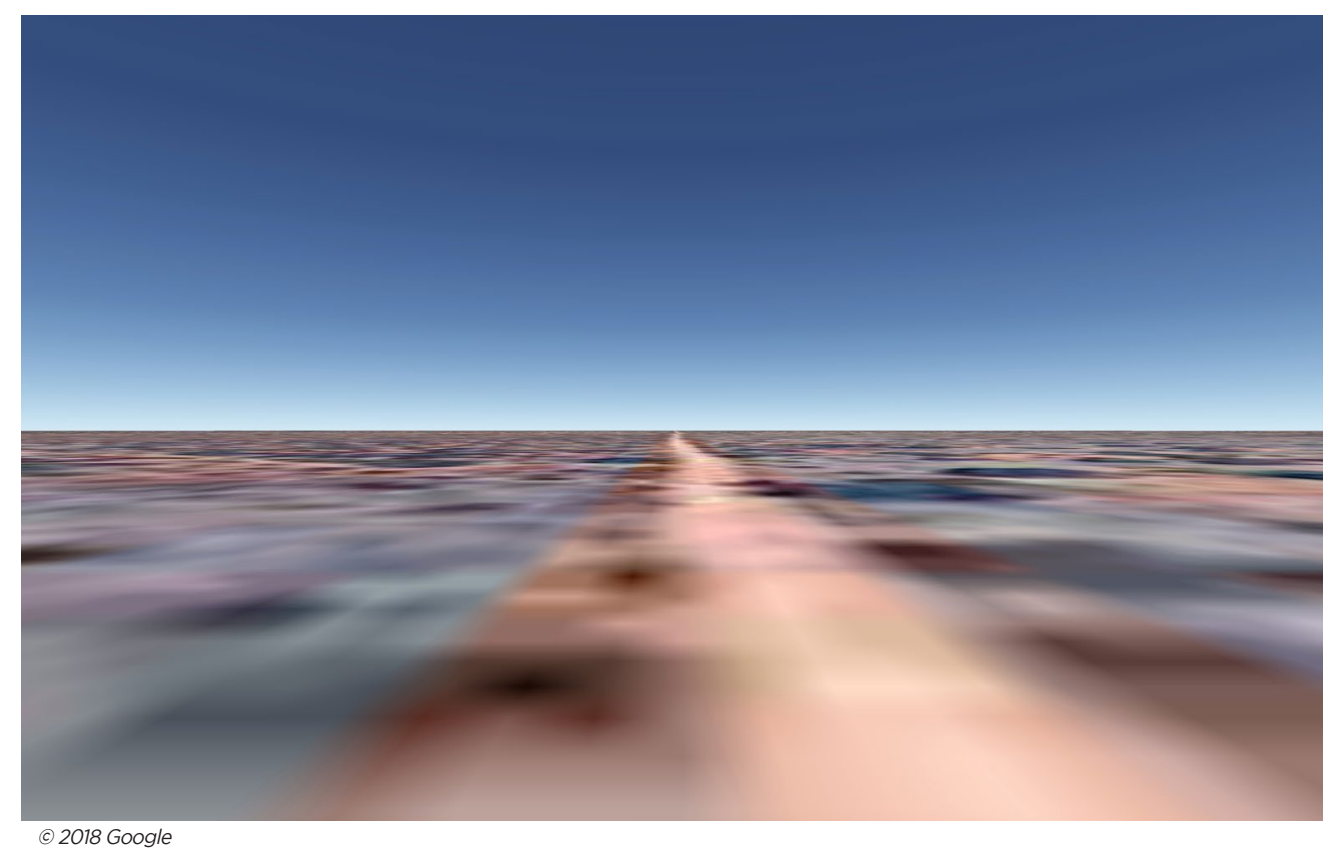


Borders in Globalization Review | Volume 3 | Issue 1 | Fall/Winter 2021

Mutter, "Murs frontières et virtualité"

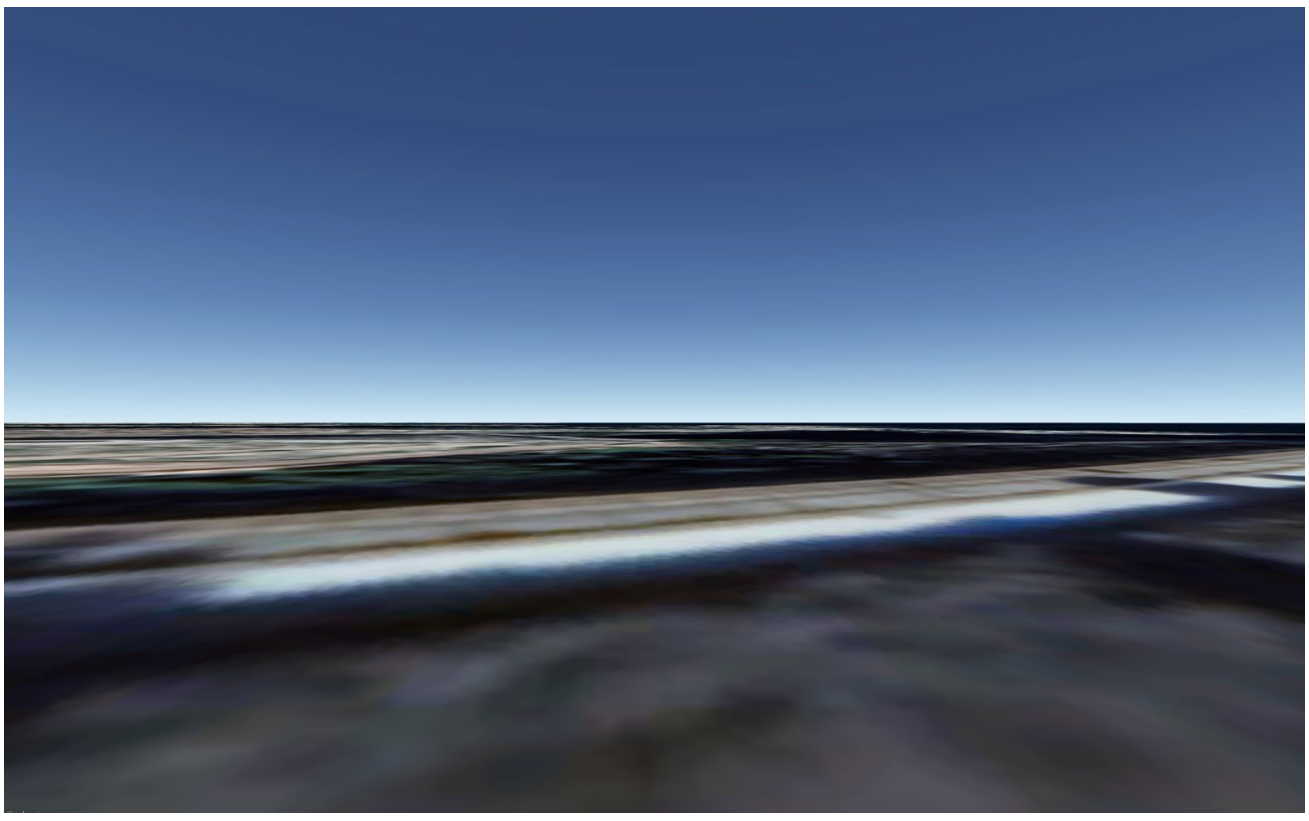

(c) 2018 Google

le regard de l'humanité sur le monde (Coulais 2015, 177) qui a été entièrement transformé. L'enjeu de ce type de représentations nécessite qu'aujourd'hui on les considère en tant qu'images qui font partie d'un dispositif visuel précis. L'utilisation militaire n'a de cesse de redéfinir cet espace de vision, car « ce nouveau régime de domination »(Deleuze 2018, 12)1 tel que l'a qualifié Gilles Deleuze, produit avant tout une suprématie stratégique. Hannah Arendt faisait part de son scepticisme quant aux progrès et aux politiques expansionnistes américaines en pleine guerre froide. Que devient l'homme en s'éloignant de la Terre ? La philosophe mettait en garde contre la fuite de l'homme face à sa condition, fuir " la terre pour l'univers » et fuir «le monde pour le Moi » (Arendt 1992, 39). ${ }^{2}$ Le premier aspect de cette analyse renvoie à la " conquête de l'espace » et à la volonté de l'homme d'atteindre le cosmos. Prenant comme exemple l'envoi d'un satellite en orbite en 1957, Arendt considère alors que le plus grand exploit de la science moderne est bien cette conquête de l'espace, mais qu'elle représente un véritable danger pour l'homme. Là où l'homme se voit conquérant de l'espace et du cosmos, se développe par corrélation une puissance et une domination techniques, annonciatrices de sa propre destruction.

Initialement, le logiciel Google Earth était connu sous le nom de «Earth Viewer » et commercialisé par la société Keyhole, dont le fond d'investissement In-Q-Tel était géré par la CIA. Aujourd'hui, qui mieux que Google peut symboliser le phénomène de globalisation et de contrôle? Google Earth, l'un de ses logiciels gratuits les plus populaires, apparu en 2005, permet à tout utilisateur connecté à internet de parcourir le monde en quelques instants. Le message est clair : il est " le globe terrestre le plus détaillé au monde » (slogan en page d'accueil du logiciel : https://www.google. $\mathrm{fr} /$ intl/fr/earth/). Google Earth s'adresse à tout utilisateur en lui proposant, entre autres, les actions suivantes : " créez des histoires et des cartes », " trouvez un lieu qui vous plaít », « partez à l'aventure », « racontez votre histoire ». Google Earth ou, traduit littéralement, Google Terre. J'emprunte ici l'explication que propose Marc Dorrian du mot Google dans son article "sur Google Earth », dans lequel il rappelle que Google tire son nom du mot gogol, nombre gargantuesque représenté par un nombre entier qui s'écrit avec le chiffre 1 suivi de 100 zéros. Google Earth ou « le gigantisme de la terre ».

" Si nous vivons, comme d'aucuns le prétendent, à l'âge de l'image aérienne, alors Google Earth en est l'un des principaux responsables. Aujourd'hui, la vue aérienne (l'image de partout) semble être partout, et on peut affirmer que Google Earth est sans doute la manifestation la plus importante et la plus stimulante de cet appétit contemporain et vorace pour les vues du ciel. De plus en plus, les relations au sol sont induites de manières complexes depuis le ciel ; une situation que Google Earth, grâce à la gigantesque disponibilité des images qu'il propose, a largement contribué à créer. Très appréciée pour ses effets spectaculaires et saisissants, la vue aérienne s'est véritablement imposée comme un élément récurrent de la culture visuelle populaire, des formes de supports et des installations touristiques » (Dorrian 2012, 230-231).

Comme le souligne ici l'auteur, il est vrai d'affirmer qu'aujourd'hui la vue aérienne est une imagerie qui fait partie intégrante de notre quotidien. Mais utilisée via l'interface Google Earth, elle constitue à mon sens un outil ludique et de distraction, dont l'unique finalité est de faire croire au spectateur qu'il est ce regard souverain et observateur sur le monde, qu'il est « un cyber-touriste » 
(Dorrian 2012, 232), comme le qualifie Dorrian. De fait, à la différence des images aériennes véhiculées au début du XXe siècle, que nous montrent les vues aériennes de Google Earth?

Lorsque nous naviguons dans le logiciel de Google, nous pouvons zoomer et dé-zoomer à l'infini sur le globe terrestre. Mais qu'est-ce qui apparaît, en premier lieu, lorsque nous ouvrons le logiciel ? Sur l'écran apparaít une petite main blanche, qui fait office de curseur spatial et qui nous permet de faire tourner la terre, un " simulacre numérique » (Dorrian 2012, 233) (d'ailleurs, en 2018, Google Maps a troqué son planisphère contre un globe terrestre en 3D), comme on ferait tourner un globe entre nos mains. Nous nous situons à une altitude de 11000 kilomètres environ, ne sachant pas très bien où nous sommes, flottant dans l'espace. À droite de l'écran, un curseur nous permet de zoomer et dé-zoomer sur le globe. Ce qu'il est intéressant de constater, c'est qu'à une altitude de 68 mètres, le point de vue vertical se redresse pour adopter le point de vue du piéton, « l'affichage au niveau du sol » comme indiqué sur l'écran.

«Google Earth nous présente une image dénuée de toute aura, dans laquelle le « magnifique joyau » des images d'apollo se trouve fragmenté (dans l'espace et dans le temps) en une panoplie d'ensembles de données géospatiales produites par les satellites en orbite et les dispositifs de capture d'image de capacité inférieure, pour être ensuite recousues numériquement, et pour enfin reconstituer l'image dans sa totalité » (Dorrian 2012, 234).

D'après l'analyse que propose Marc Dorrian des images véhiculées par l'interface du logiciel, on peut alors avancer l'affirmation suivante : la logique visuelle de Google Earth est la même que celle de la guerre depuis l'apparition des visuels de la première guerre du Golfe. En utilisant Google Earth, nous laissons libre cours à notre puissance d'immixtion dans le domaine tant public que privé tout comme l'avidité de la puissance militaire peut avoir accès au territoire qu'elle convoite. Par ailleurs, dans les deux cas, nous sommes face à une vision fragmentée de ce que nous voyons. Les images qui composent le globe terrestre proposées par Google Earth, telle une sphère éclatée en milliers de pixels et de strates visuelles, sont composées d'une multitude d'informations et de données qui sont elles-mêmes assemblées, reconstituées,

" recousues », afin de ne montrer qu'une infime partie d'un ensemble, à savoir l'image qui sera proposée au spectateur. Dans le champ visuel de la guerre et surtout dans les conflits contemporains, on peut constater un phénomène d'éclatement quant aux images diffusées et que l'on veut bien nous montrer des conflits. Tout comme les visuels de la guerre du Golfe sont devenus la surface de l'écran télévisuel, les images reconstituées deviennent la « surface numérique » (Dorrian 2012, 235) de Google.

\section{Des lignes frontières}

Mais avec des logiciels tels que Google Earth, peut-on aujourd'hui envisager la frontière comme un espace entièrement virtuel, dont les limites seraient elles-mêmes indéfinissables ? Étymologiquement, la frontière vient du provençal "fronteira ", l'endroit où deux armées se font front, s'affrontent. L'histoire de la séparation des hommes par des murs semble donc être indissociable de la guerre, et il était à mon sens nécessaire de penser la représentation virtuelle des murs frontières à la lumière de la représentation de la guerre. C'est de cette réflexion que

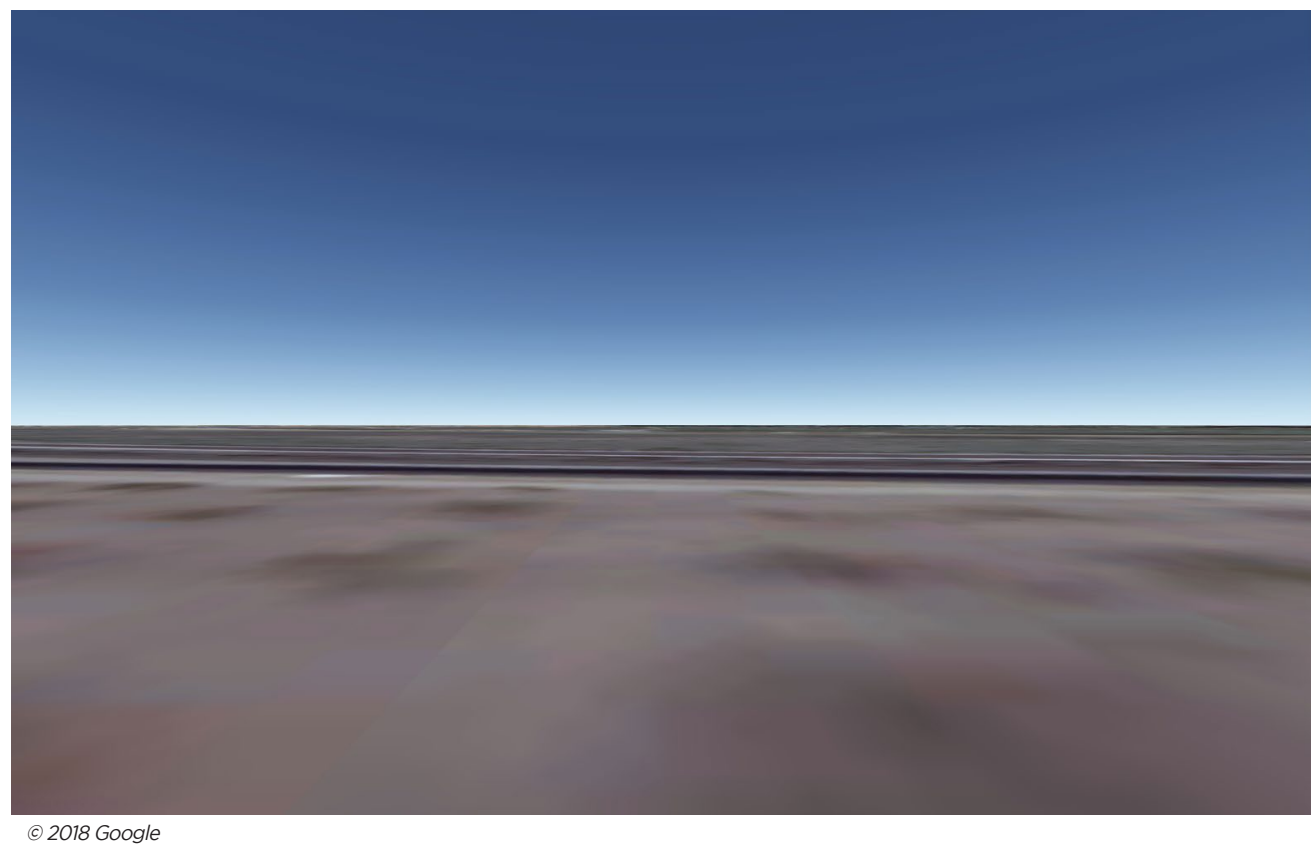


Borders in Globalization Review | Volume 3 | Issue 1 | Fall/Winter 2021

Mutter, "Murs frontières et virtualité"

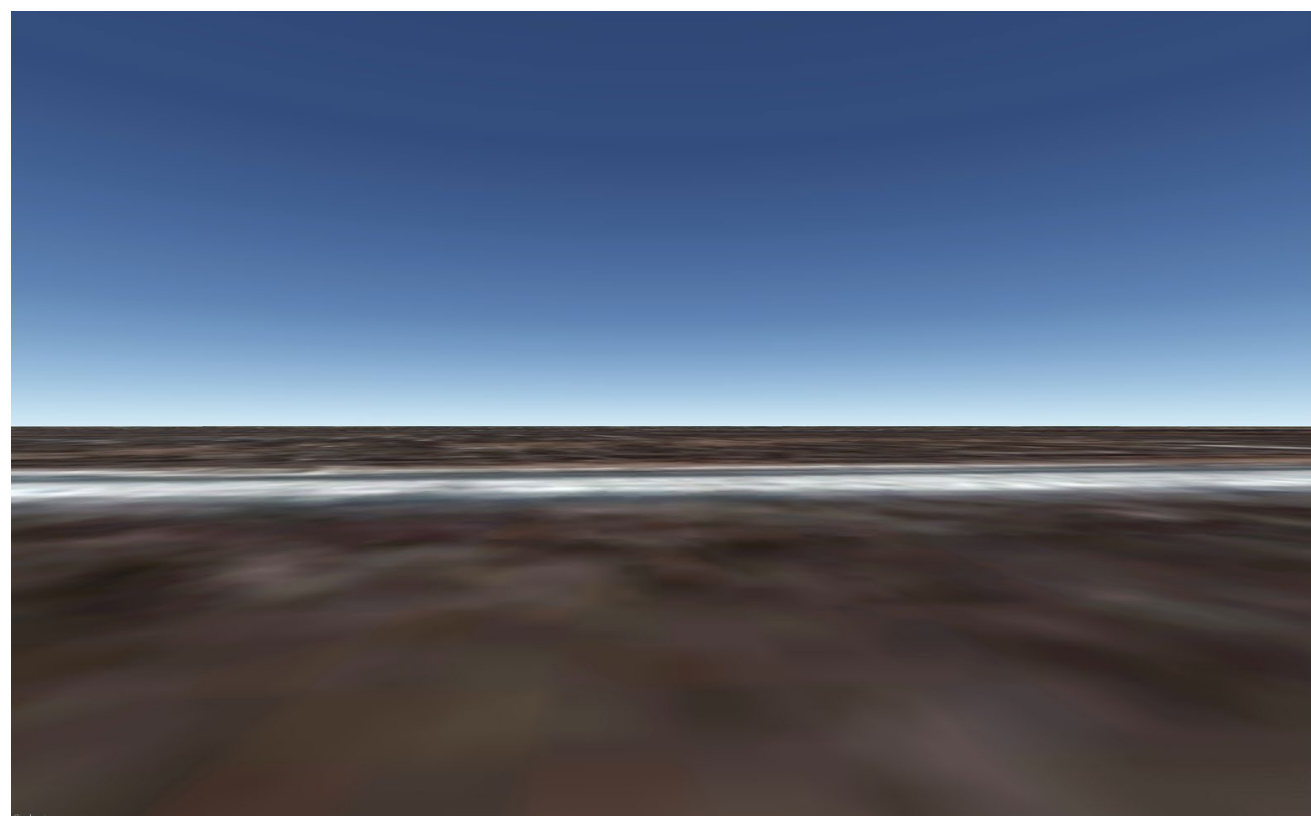

(c) 2018 Google

découle mon projet «Lines », qui a entièrement été réalisé sur le logiciel Google Earth, où j'ai recherché et archivé trente-neuf murs frontières qui existent encore aujourd'hui dans le monde sur la soixantaine déjà construits ou en cours de construction. Projet qui questionne l'ambiguité quant à la confrontation entre la réalité vue à travers un logiciel et la représentation virtuelle du réel, j'ai très vite été confrontée à la limite propre de la représentation des technologies numériques n'obtenant, pour seule représentation de murs, que des lignes abstraites. En faisant se rejoindre la ligne d'horizon et la ligne de la frontière, ces images produisent un éloignement continu de la frontière et de loin, elles semblent être un trait discontinu tout comme l'est le trait discontinu sur une carte.

Avec le point de vue du piéton, on se rend vite compte que l'image est très loin de la réalité : elle est entièrement virtuelle, pixellisée et standardisée. Seules changent, en fonction des régions du monde, la couleur des pixels utilisés, stylisation de plus qui tente de coller à la réalité. Bien loin des images aériennes satellitaires habituellement diffusées via Google Earth, on assiste à une simplification évidente du champ du réel : toutes les images se ressemblent et paraissent sortir du même jeu vidéo et notre œil semble s'être aujourd'hui habitué à une telle logique de production des images.

Les images de Google Earth sont intéressantes ici car on est dans une mise à distance totale de la frontière, qui se traduit également par une mise à distance des corps, qui sont inexistants. Ces images permettent de mettre à distance la réalité en brouillant les informations, elles ne coincident pas avec ce que l'on voit quotidiennement. Ce sont des images qui ne sont pas là pour informer, elles tiennent à distance l'observateur puisqu'elles ne signifient, en apparence, rien. Avec la multiplication des points de vue et des perspectives par la prolifération des modes de diffusion et de fabrication des images, il en va également de la multiplication des représentations de la frontière qui devient elle-même une représentation aléatoire. On sait dès lors quand elle commence, mais on ne sait pas où ni comment elle va finir.

\section{Liste des murs frontières représentés dans le projet « Lines »:}

- Afghanistan/Ouzbékistan

- Afrique du Sud/Mozambique

- Afrique du Sud/Zimbabwe

- Algérie/Maroc

- Bostwana/Zimbabwe

- Bulgarie/Turquie

- Espagne-Ceuta/Maroc

- Corée du Nord/Corée du Sud

- Egypte/Bande de Gaza

- France-Calais/Royaume-Uni

- Bande de Gaza/Israël

- Grèce/Turquie

- Inde/Bangladesh

- Inde/Birmanie

- Inde/Pakistan-Cachemire

- Irak/Turquie

- Iran/Pakistan

- Israël/Cisjordanie

- Israël/Egypte

- Israël/Liban

- Israël/Syrie

- Jordanie/Irak

- Kenya/Somalie

- Libye/Tunisie

- Macédoine/Grèce

- Malaisie/Thailande 
- Maroc/Sahara Occidental

- Espagne-Melilla/Maroc

- Mexique/Etats-Unis

- Oman/Emirats Arabes Unis

- Oman/Yémen

\section{Notes}

1. Article paru dans L'Autre journal $n^{\circ} 1$, mai 1990 , repris sous le titre "Post-scriptum sur les sociétés de contrôle » in Pourparlers. 1972-1990, éditions de Minuit, 1990, p. 247.

2. Traduction de The Human Condition, London, Chicago, University of Chicago Press, 1958 ; première traduction française en 1961, cité par Bernard Debbieux, « Les spatialités dans l'œuvre d'Hannah Arendt ", Cybergeo : European Journal of Geography [En línea], Epistemología, Historia de la Geografía, Didáctica, documento 672, Publicado el 02 abril 2014. https://doi.org/10.4000/ cybergeo.26277

\section{Works Cited}

Arendt, Hannah. 1992. Condition de l'homme moderne, Pocket, Paris.

Aubout, Mickaël. 2011. «Le milieu aérien, acteur et objet du renseignement », dans Hérodote, 1(140).

Coulais, Jean-François. 2015. Images virtuelles et horizons du regard, MetisPresses, VuesDensemble, Genève.

Deleuze, Gilles. 2018. "Les sociétés de contrôle », in EcoRev, n46, 2018(1). https://doi.org/10.3917/ecorev.046.0005

Dorrian, Marc. 2012. «Sur Google Earth », in Seize études pour une histoire culturelle: vues aériennes, sous la direction de Mark Dorrian et Frédéric Pousin, Metispresses, Genève.

Frizot, Michel. 2001. « Une autre photographie, les nouveaux points de vue », dans Nouvelle histoire de la photographie, sous la direction de Michel Frizot, Larousse, Paris.

Tzu, Sun. 2008. L'art de la guerre. Flammarion, Paris. 